\title{
Voluntary and anonymous reporting of medication errors in patients admitted to the Department of Pediatrics
}

\author{
José M. Ceriani Cernadas, M.D. ${ }^{a, b}$, Luz Bogado, B.N. ${ }^{a}$, Fidencia Espinola Rolón, B.N. \\ and María F. Galletti, M.D. ${ }^{b}$
}

"Any man can make mistakes,
but only an foolish persists in his error".

Cicero

$(106 \mathrm{BC}-43 \mathrm{BC})$

\begin{abstract}
Objective. To assess reports of medication errors in hospitalized children to establish their frequency, causes, and adverse events(AEs).

Methods. Prospective study of medication errors reported at the Neonatal Intensive Care Unit (NICU), Pediatric Clinic (PC), and Pediatric intensive Care Unit (PICU). Ambulatory Pediatrics reports and incomplete data were excluded. Related variables were evaluated.

Results. Out of 989 errors reported in Department of Pediatrics, 401 (41\%) corresponded to medication errors. Of these, $353(88 \%)$ reached patients and $48(12 \%)$ were quasi-errors; $42 \%$ occurred in the morning; $24 \%$, in the afternoon; $17 \%$, in the morning and afternoon, and $17 \%$, in the night; differences were notsignificant $(p=0.18)$. Dosing errors were the most common ones, 118 (33.4\%). In total, 160 reports $(45 \%)$ corresponded to prescription errors; 149 (42\%), to administration errors; and $44(2.5 \%)$, to dispensing errors. Dosing errors were more common in prescriptions $(p<0.05)$. The highest number of errors was reported at the NICU, 179 (50\%); compared to 91 (25.5\%) at the PC and $83(24.5 \%)$ at the PICU. A total of 91 AEs were reported $(22.5 \%)$; most were mild, $53(58 \%)$, or moderate, $31(34 \%)$. The NICU reported 53 AEs (58\%); the PICU, $25(27 \%)$; and the DCP, $18(19.7 \%)$. No deaths occurred. Conclusion. The rate of medication errors reported in hospitalized children was $41 \%$. Dosing errors were the most common ones. A total of 91 AEs were reported; most were mild (58\%); the highest number was reported at the NICU $(58 \%)$.

Key words: pediatrics, medication errors, selfreporting, hospitalization, adverse events.
\end{abstract}

http:/ / dx.doi.org/10.5546/ aap.2019.eng.e592

To cite: Ceriani Cernadas JM, Bogado L, Espínola Rolón F, Galletti MF. Voluntary and anonymous reporting of medication errors in patients admitted to the Department of Pediatrics. Arch Argent Pediatr 2019;117(6):e592-e597.

\section{INTRODUCTION}

Errors are common and happen on a daily basis in every activity carried out by human beings. In medicine, they are caused, in particular, by two factors. The most common factor refers to deficiencies in the hospital system that take place in most actions related to errors in patient care. Higher risk systems at the hospital include patient handoff, change of nurse shifts, communication among the members of hospital teams, communication with the patient at the time of discharge, operating room checklists before a surgery, patient identification, pharmacy drug supply, among others.

The second factor is related to errors made by people, and these are mainly due to lack of attention, stress, burnout syndrome, rules or regulations, and knowledge deficits. ${ }^{1}$ Even when errors are part of the human condition, there is no justification for them to occur in health care as a result of individuals. ${ }^{2}$

In the past 25 years or so, multiple measures were taken to achieve adequate patient safety, but an alarming quantity of medical errors is still prevalent. In the United States of America (USA), more than 200000 patients die every year due to errors, although recent data indicate that the annual mortality rate has jumped to more than 300000 patients. ${ }^{3,4}$

To reduce errors, it is necessary to change the traditional medical error culture, which encourages hiding 
the error and punishing those responsible for it. Unfortunately, such culture still predominates in most health care facilities, ${ }^{5}$ at least in our country, and it is worth noting that communication among the members of a department is inadequate. An effective communication is one of the most relevant measures that can be taken to reduce errors and adverse events (AEs).

Medication errors are the most common type in hospitalized children, ${ }^{6-9}$ in particular in the Neonatal Intensive Care Unit (NICU). ${ }^{10,11}$ The rate of AEs in newborn infants and children is three times higher than in adults. ${ }^{8}$ Errors and AEs are usually the result of failures in the medication process and of health care providers' neglect, usually due to attention deficits. ${ }^{12-15}$ The analysis of error causes and the strategies aimed at preventing them help to reinforce the culture of safety in hospital care ${ }^{16}$ The medication process is made up of four stages: prescription, carried out by physicians; administration, carried out by nurses; and transcription and supply, both in charge of pharmacists.

Voluntary error reports are implemented among the health care providers of medical facilities and usually include information about where the error occurred, the type of error and, less commonly, resulting AEs. The main benefit of these reports is to help establishing a change in error culture so as to prevent punitive actions and encourage learning from errors so that they do not happen again. ${ }^{17,18}$

However, reported information is usually incomplete and non-systematic because errors tend to be hidden. Unfortunately, some facilities commonly report mild and moderate events only, not severe ones. ${ }^{19}$ Therefore, error reporting systems are not very helpful to determine the extent of safety in hospital care. Notwithstanding this, the increase in error reporting at a health care facility is a good indication that the culture may be improving and, therefore, of a better patient safety because such communication means that health care providers are not so afraid of reporting errors and, this way, punishment will also reduce progressively. ${ }^{19-25}$

The objective of this study was to assess the reports of medication errors in hospitalized children to establish their frequency, causes, and AEs with potential consequences on patients.

\section{POPULATION AND METHODS}

This was an observational, prospective study to assess the reports of medication errors in patients admitted to the Department of Pediatrics of Hospital Italiano de Buenos Aires between January $2^{\text {nd }}, 2016$ and December $30^{\text {th }}, 2017$. Data were collected from the anonymous and voluntary electronic error reporting system, where reports were electronically entered using the hospital's intranet, which could only be accessed by the hospital's health care providers.

Licensed nurses who were members of the Patient Safety Committee collected all anonymous error reports in a prospective manner and then selected those who had taken place at the Department of Pediatrics.

Medication errors reported at the Pediatric Clinical (PC), the Pediatric Intensive Care Unit (PICU), and the NICU for patients from birth to 16 years old were included. Reports of medication quasi-errors were also included, i.e., errors that did not reach the patient.

Reports made at outpatient pediatric offices or with incomplete or missing data were excluded.

At Hospital Italiano, drugs are prepared by the Pharmacy Department and then supplied to each area. Prescription at each area depended on physicians; and nurses, together with pharmacists, were in charge of preparing the drugs indicated in the prescription. This took place in a closed room, free of noise and with no access to mobile phones or telephones, so that they would not be distracted.

The study was approved by the Research Ethics Committee of Hospital Italiano de Buenos Aires (no. 3505). It was defined that it was not necessary to obtain informed consent because reports were anonymous and none included patients' names.

The following outcome measures of reported errors were evaluated

Error origin: Prescription by physicians, administration by nurses, and drug supply by pharmacists.

Type of medication errors: Dosing error, prescription of the wrong drug, error in drug dilution, drug omission, error in frequency of administration, wrong patient, error in route of administration, error in drug concentration, wrong drug presentation, dispensing the wrong drug, administering the wrong drug, error in the rate of intravenous infusion.

The day when the error occurred: Mondays through Fridays or Saturdays and Sundays.

Nurse shift: In the morning, afternoon or night.

Area of the Department of Pediatrics: Errors were reported at the PC, PICU or NICU. 
Frequency of AEs and harmful effects: Mild (no test required), moderate (requiring lab tests and greater care) or severe (requiring tests and special management).

\section{Statistical analysis}

For each outcome measure mentioned here, data were described using discrete quantitative outcome measures, with frequency and percentage values. Errors were described as absolute and relative frequency. A statistical analysis was done to compare the proportion of errors by origin (prescription, administration) and nurse shift. AEs and their potential harmful effects were also described. In all cases, a $p<0.05$ difference was considered statistically significant. The $\chi^{2}$ test or Fisher's exact test were used for low expected frequencies.

\section{RESULTS}

A total of 4986 errors were reported at the hospital; 989 (20\%) occurred at the Department of Pediatrics, and $401(41 \%)$ corresponded to medication errors, which were the most common ones. Of these, $353(88 \%)$ errors reached the patients, whereas $48(12 \%)$ were quasierrors. Dosing errors were the most common type, $118(33.4 \%)$, followed by drug omission, $47(13.3 \%)$ (Table 1). Seventy-nine dosing errors $(67 \%)$ were related to drug prescription and $39(33 \%)$, to drug administration $(p=0.07)$; no dispensing errors were reported. A total of 283 reports ( $80 \%$ ) were made on Mondays through Fridays, whereas $70(20 \%)$ were made on the weekend. Also, 149 reports $(37 \%)$ were made in the morning shift; $84(21 \%)$, in the afternoon shift; $60(15 \%)$, in the morning and afternoon shift (Saturdays and Sundays); and $60(15 \%)$, in the night shift; the differences were not significant, $p=0.8$. The most common errors were prescription errors, which accounted for 165 reports $(46.7 \%) ; 144(40.8 \%)$ accounted for administration errors; and 44 (12.5\%), dispensing errors. Dosing errors were significantly more frequently made in prescriptions $(p<0.05)$. The highest number of errors were reported at the NICU, $179(50 \%)$; compared to $91(25.5 \%)$ at the PC and $83(24.5 \%)$ at the PICU. A total of 91 AEs $(22.5 \%)$ were reported; $54(58 \%)$ were mild; $32(34 \%)$, moderate; and $5(1.4 \%)$, severe. The NICU reported 53 AEs (58\%); the PICU, 20 (22\%); and the PC, 18 (19.7\%); the difference was not significant, $p=0.09$. No medication errorassociated mortality was observed. The analysis of reported quasi-errors showed that $23(48 \%)$ were potentially mild; $19(39.5 \%)$, potentially moderate; and $6(12.5 \%)$, potentially severe.

\section{DISCUSSION}

This study assessed multiple outcome measures associated with voluntary reporting of medication errors in patients admitted to the Department of Pediatrics of Hospital Italiano de Buenos Aires.

The voluntary error reporting initiative at health care facilities has reached a greater prevalence in the beginning of the century; its main objective is to bring about a change in the traditional patient safety culture, which, based on prejudice, considers that errors are only caused by human beings and that, therefore, they should be punished.

Still today, such culture persists in many health care facilities. The rate of publications about error reporting has increased mostly in developed countries, especially the USA and Western Europe, where health care systems prioritize health care safety. ${ }^{3,5,19,22-26}$

TABLE 1. Type of error

\begin{tabular}{|c|c|c|}
\hline & $\mathbf{N}$ & $\%$ \\
\hline Dosing error & 118 & 33.4 \\
\hline Drug omission & 47 & 13.3 \\
\hline Prescription of the wrong drug & 36 & 10.2 \\
\hline Error in drug dilution & 27 & 7.7 \\
\hline Error in frequency of administration & 24 & 6.8 \\
\hline Wrong patient & 23 & 6.5 \\
\hline Dispensing the wrong drug & 22 & 6.2 \\
\hline Administering the wrong drug & 17 & 4.8 \\
\hline Error in route of administration & 13 & 3.7 \\
\hline Miscellaneous & 26 & 4.9 \\
\hline Total & 353 & 96 \\
\hline
\end{tabular}


It is worth noting that published studies correspond mostly to errors reported in adults, whereas only a few have been conducted in pediatric patients. ${ }^{16,18}$ However, several publications related to reports in pediatrics have mostly approached medication errors and their potential consequences. ${ }^{6-12,14,27-30}$ Still, in Latin American countries, reporting studies are very scarce compared to developed countries. So much that, to our knowledge, this is the first Argentine study that focused exclusively on reports of medication errors in hospitalized children.

Error reporting is very helpful to detect system failures, quasi-errors, and AEs but, as pointed out, it would be particularly useful to change the patient safety culture in health care facilities. Voluntary reporting is only one of the multiple pieces that are required at hospitals to reduce and detect errors and AEs in order to improve patient safety..$^{21,23-25,30-32}$

As observed in this study, medication errors are most commonly reported in a voluntary manner. Most likely, these errors are more easily detected in hospitalized children, by both health care providers and patients' parents, who are usually the first ones to notice them. ${ }^{33,34}$

AEs are more common in medication errors than in other types of errors; in our study they also occurred more often than usual. Likewise, some studies have observed that AEs resulting from medication errors account for a much higher risk than other errors. ${ }^{25}$

Spontaneous and voluntary reporting systems for health care providers are expected to report any error and all AEs. An open, non-punitive, strictly confidential approach will promote voluntary reporting. However, at health care facilities, several health care providers still fear disciplinary or legal actions against those who report errors. Because of this, most reports should be anonymous and voluntary so that there are less chances of punishment on reporting health care providers.

The results of this study were mostly similar to those observed in different investigations done in other countries $6,9,10,14,17,29,31$ and, although the frequency may differ, many consistencies can be seen. Among others, it is worth mentioning that the rate of prescription errors was higher than that of administration errors, which has been described in most published studies, both in adults and children. ${ }^{29,31}$ The same is true for the fact that dosing errors were the most common type of errors.
In addition, more than half of errors were reported at the NICU, which was also consistent with other studies. ${ }^{6,7,9}$ This is mostly due to the intricate process entailed by the preparation of drugs for newborn infants which, among other things, requires multiple dilutions, especially in the case of very small preterm infants. ${ }^{10,22,30,31}$

Moreover, the studies that analyzed errors reported in hospitalized children at the Department of Pediatrics found that the rate of AEs at the NICU was higher than the rest ${ }^{8,12,29,34-}$ ${ }^{36}$ and this was also observed in our study, where more than half of all AEs occurred at the NICU. The 3 severe AEs that occurred at the NICU corresponded to dosing errors: 1 in the physician's prescription and 2 due to drug dilution mistakes made by nurses.

Even with the several limitations pointed out in relation to anonymous and voluntary reports of errors in patient safety, the data collected in this study have been very useful to establish the frequency of reported medication errors and also accurately detect AEs and their consequences. It was also possible to perform root-cause analyses applied to severe or sentinel AEs, and this enabled the identification of failures in hospital systems and health care providers in charge of patient care. In this way, a greater knowledge was obtained about error causes and reasons.

We also included reports of errors that did not reach the patient, known as "quasi-errors," and this was very helpful to detect how they occurred and, thus, the barriers that prevented them from reaching the patients. This is a very relevant aspect because error prevention barriers are one of the most important measures in patient safety to reduce or avoid AEs. ${ }^{9,10,25,27,32-35}$

\section{CONCLUSION}

The primary objective of this study was met: assessing medication error reports in terms of error frequency, causes, and resulting AEs and consequences. Out of all error types, dosing errors were the most common ones, three times more frequent than the second leading type. Mild AEs predominated and accounted for two-thirds of all AEs. The highest rate of medication errors at the Department of Pediatrics was reported at the NICU, as well as the highest AE rate. Most reports were made by physicians, and prescription errors were more common than administration errors. No errors were reported in relation to deceased patients. 


\section{REFERENCES}

1. Ceriani Cernadas JM. El error en medicina: reflexiones acerca de sus causas y sobre la necesidad de una actitud más crítica en nuestra profesión. Arch Argent Pediatr. 2001; 99(6):522-6.

2. Reason J. Human error: models and management. BMJ. 2000; 320(7237):768-70.

3. James JT. A new, evidence-based estimate of patient harms associated with hospital care. J Patient Saf. 2013; 9(3):122-8.

4. Kavanagh KT, Saman DM, Bartel RB, Westerman K. Estimating Hospital-Related Deaths Due to Medical Error: a perspective from patient advocates. J Patient Saf. 2017; 13(1):1-5.

5. Verbakel N, Langelaa M, Verheij T, Wagner C, et al. Improving patient safety culture in primary care: a systematic review. J Patient Saf. 2016; 12(3):152-8.

6. Ross LM, Wallace J, Paton JY. Medication errors in a paediatric teaching hospital in the UK: five years operational experience. Arch Dis Child. 2000; 83(6):492-7.

7. Stucky ER, American Academy of Pediatrics Committee on Drugs, American Academy of Pediatrics Committee on Hospital Care. Prevention of medication errors in the pediatric inpatient setting. Pediatrics. 2003; 112(2):431-6.

8. Sharek PJ, Classen D. The incidence of adverse events and medical error in pediatrics. PediatrClin North Am. 2006; 53(6):1067-77.

9. Otero P, Leyton A, Mariani G, CerianiCernadas JM, Patient Safety Committee. Medication errors in pediatricinpatients: prevalence and results of a prevention program. Pediatrics. 2008; 122(3):e737-43.

10. Lucas AJ. Improving medication safety in a neonatal intensive care unit. Am J Health Syst Pharm. 2004; 61(1):33-7.

11. Ceriani Cernadas JM. El desafío de reducir los errores de medicación en las Unidades de Cuidado Intensivo Neonatal. Arch Argent Pediatr. 2016; 114(5):394-5.

12. Chedoe I, Molendijk H, Hospes W, Van den Heuvel ER, et al. The effect of a multifaceted educational intervention on medication preparation and administration errors in neonatal intensive care. Arch Dis Child Fetal Neonatal Ed. 2012; 97(6):F449-55.

13. Fahrenkopf AM, Sectish TC, Barger LK, Sharek PJ, et al. Rates of medication errors among depressed and burnt-out residents: prospective cohort study. BMJ. 2008; 336(7642);488-91.

14. Lyren A, Brilli RJ, Zieker K, Marino M, et al. Children's Hospitals' Solutions for Patient Safety Collaborative Impact on Hospital-Acquired Harm. Pediatrics. 2017; 140(3):e20163494.

15. Fortescue E, Kaushal R, Landrigan C, McKenna K, et al. Prioritizing strategies for preventing medication errors and adversedrugeventsinpediatricinpatients.Pediatrics.2003;111 (4 Pt 1):722-9.

16. Bradley EH, Brewster AL, McNatt Z, Linnanderet E, et al. How guiding coalitions promote positive culture change in hospitals: a longitudinal mixed methods interventional study. BMJ QualSaf. 2018; 27(3):218-25.

17. Beck JB, McGrath C, Toncray K, Rooholamini SN. Failure Is an Option: Using Errors as Teaching Opportunities. Pediatrics. 2018; 141(3):e20174222.

18. Burlison JD, Quillivan RR, Kath LM, Zhou Y, et al. A Multilevel Analysis of U.S. Hospital Patient Safety Culture
Relationships with Perceptions of Voluntary Event Reporting. J Patient Saf. 2016. [Epub ahead of print].

19. Sari A, Sheldon TA, Cracknell A, Turnbull A. Sensitivity of routine system for reporting patient safety incidents in an NHS hospital: retrospective patient case note review. BMJ. 2007; 334(7584):79.

20. Shojania KG. The frustrating case of incident-reporting systems. QualSaf Health Care. 2008; 17(6):400-2.

21. Vincent C. Incident reporting and patient safety. BMJ. 2007; 334(7584):51.

22. Suresh G, Horbar JD, Plsek P, Gray J, et al. Voluntary anonymous reporting of medical errors for neonatal intensive care. Pediatrics. 2004; 113(6):1609-18.

23. Ceriani Cernadas JM, Otero P, Leyton A, Mariani G, et al. Anonymous Reporting of Medical Errors. Another Strategy To Promote Patient Safety. E-PAS. 2006; 59:3562.284.

24. Mitchell L, Schuster A, Smith K, Pronovost P, et al. Patient safety reporting: a qualitative study of thoughts and perceptions of experts 15 years after 'To Err is Human'. BMJ QualSaf. 2016; 25(2):92-9.

25. Aranaz-Andrés JM, Aibar-Remón C, Limón-Ramírez R, Amarilla A, et al. Prevalence of adverse events in the hospitals of five Latin American countries: results of the 'Iberoamerican Study of Adverse Events' (IBEAS). BMJ Qualsaf. 2011; 20(12):1043-51.

26. Berry JC, Davis JT, Bartman T, Hafer C, et al. Improved safety culture and teamwork climate are associated with decreases in patient harm and hospital mortality across a hospital system. J Patient Saf. 2016. [Epub ahead of print].

27. Worthen WM. After the Medical Error. JAMA. 2017; 317(17):1763-4.

28. Howell AM, Burns EM, Bouras G, Donaldson LJ, et al. Can patient safety incident reports be used to compare hospital safety? Results from a quantitative analysis of the English National Reporting and Learning System Data. PLoS One. 2015; 10(12):e144107.

29. Dedefo MG, Mitike AH, Angamo MT. Incidence and determinants of medication errors and adverse drug events among hospitalized children in West Ethiopia. BMC Pediatr. 2016; 16:81.

30. Adelman JS, Aschner JL, Schechter CB, Angert RM, et al. Evaluating Serial Strategies for Preventing Wrong-Patient Orders in the NICU. Pediatrics. 2017; 139(5):e20162863.

31. Kozer E, Berkovitch M, Koren G. Medication Errors in Children. PediatrClin North Am. 2006; 53(6):1155-68.

32. Steering Committee on Quality Improvement and Management and Committee on Hospital Care. Policy Statement-Principles of Pediatric Patient Safety: Reducing Harm Due to Medical Care. Pediatrics. 2011;127(6):1199-210.

33. Khan A, Coffey M, Litterer KP, Baird JD, et al. Families as Partners in Hospital Error and Adverse Event Surveillance. JAMA Pediatr. 2017; 171(4):372-81.

34. Khan A, Furtak SF, Melvin P, Rogers JE, et al. ParentReported Errors and Adverse Events in Hospitalized Children. JAMA Pediatr. 2016; 170(4):e154608.

35. Stockwell DC, Landrigan CP, Toomey S, Loren SS, et al. Adverse Events in Hospitalized Pediatric Patients. Pediatrics. 2018; 142(2):e20173360.

36. Gates PJ, Meyerson SA, Baysari MT, Lehmann CU, et al. Preventable Adverse Drug Events Among Inpatients: A Systematic Review. Pediatrics. 2018; 142(3):e20180805. 